\title{
COMPARAÇÃO ENTRE DOIS TIPOS DE SUSPENSÃO PASSIVA DE BARRA EM CONDIÇÕES SIMULADAS DE PISTA DE PROVA NORMALIZADA
}

\section{CRISTIANO O. PONTELLI ${ }^{1}$, MÁRIO F. MUCHERONI² ${ }^{2}$ JOSÉ M. BALTHAZAR $^{3}$, BENTO R. PONTES JÚNIOR ${ }^{4}$}

\begin{abstract}
RESUMO: Neste trabalho, foram feitas algumas análises sobre o comportamento dinâmico simulado de dois tipos de suspensões passivas das barras dos pulverizadores tracionados. Estas análises foram conduzidas em condições virtuais de pista de prova normalizada ISO 5008, com dois níveis de velocidade de deslocamento do pulverizador $\left(5 \mathrm{~km} \mathrm{~h}^{-1}\right.$ e $\left.15 \mathrm{~km} \mathrm{~h}^{-1}\right)$ e em dois perfis de pista existentes na norma (acidentada e suavizada). Foram utilizados nas simulações os softwares MATLAB $^{\circledR}$, SIMULINK $^{\circledR}$ e Visual Nastran ${ }^{\circledR}$. Os resultados mostram que a suspensão do tipo trapezoidal teve melhor desempenho em baixas frequências de excitação $(\omega<0,2 \mathrm{~Hz})$, enquanto a suspensão do tipo pêndulo simples teve melhor desempenho nas demais condições das pistas normalizadas.
\end{abstract}

PALAVRAS-CHAVE: suspensão passiva de barra, suspensão trapezoidal, suspensão pendular simples.

\section{COMPARISON BETWEEN TWO TYPES OF PASSIVE BOOM SUSPENSION UNDER SIMULATED CONDITIONS OF TRACK TEST}

\begin{abstract}
In this paper we conducted several analysis of the simulated dynamic behavior of two passive suspension types of the trailed sprayer booms. The suspension analysis was conducted under conditions of a bump track test ISO 5008, with two levels of speed $\left(5 \mathrm{~km} \mathrm{~h}^{-1}\right.$ and $\left.15 \mathrm{~km} \mathrm{~h}^{-1}\right)$ and two track profiles proposed in these standards (bumpy and smooth). It was used the simulations software MATLAB ${ }^{\circledR}$, SIMULINK ${ }^{\circledR}$ and Visual Nastran ${ }^{\circledR}$. The results obtained showed that suspensions of trapezoidal type have good performance at low frequencies input $(\omega<0,2 \mathrm{~Hz})$ while suspensions of simple pendulum type have good performance in others conditions.
\end{abstract}

KEYWORDS: passive boom suspension, twin-link suspension, simple pendulum suspension.

\section{INTRODUÇÃO}

Para aumentar a produtividade na agricultura, as culturas necessitam ser protegidas contra as doenças. Um dos mais importantes métodos para controlar as doenças é a utilização de agroquímicos, aplicados por pulverizadores. Os estudos indicam que a eficiência dos produtos químicos está altamente correlacionada com a uniformidade do padrão de distribuição (GÖHLICH, 1985). O movimento da barra do pulverizador, vertical e/ou horizontal, provoca um efeito drástico sobre o padrão de distribuição.

Os produtos químicos são aplicados através de bicos montados sobre uma barra. Essa operação é dispendiosa, e as áreas agrícolas estão ficando maiores. Atualmente, há pulverizadores de barra com comprimento de 42 m, já referenciados em 1993 (SERNEELS \& DUCATTILLON, 1993). A velocidade de caminhamento do trator normalmente varia entre 3 a $12 \mathrm{~km} \mathrm{~h}^{-1}$.

\footnotetext{
${ }^{1}$ Eng $^{\mathrm{o}}$ Mecânico, Mestre em Engenharia Mecânica, Profissional de Pesquisa, Máquinas Agrícolas Jacto S.A., pontelli@ jacto.com.br.

${ }^{2}$ Eng $^{\mathrm{o}}$ Mecânico, Doutor em Engenharia Mecânica, Prof. Doutor, Departamento de Engenharia Mecânica, USP, São Carlos - SP, mariofm@sc.usp.br.

${ }^{3}$ Eng $^{\mathrm{o}}$ Aeronáutico, Doutor em Engenharia Mecânica, Professor Titular, Departamento de Estatística, Matemática Aplicada e Computação (DEMAC), UNESP,Rio Claro - SP, jmbaltha@ rc.unesp.br.

${ }^{4}$ Eng $^{\mathrm{o}}$ Mecânico, Doutor em Engenharia Mecânica, Prof. Doutor, Departamento de Engenharia Mecânica, UNESP, Bauru - SP, bento@feb.unesp.br. 
Durante o tratamento, é fundamental aplicar uniformemente sobre o campo a dose necessária de agroquímicos. A subdosagem (quantidade menor que a necessária) não é totalmente eficaz, enquanto o excesso de aplicação leva à poluição ambiental, resíduos excessivos e gastos desnecessários.

A desigualdade na aplicação também tem consequências econômicas. Uma área com pouca aplicação de agroquímicos pode sofrer com o mau controle de ervas daninhas ou pragas, o que resulta em efeito negativo sobre o rendimento agrícola. Para maximizar o rendimento, portanto, os agricultores utilizam, em geral, um valor demasiadamente elevado de substâncias químicas (OZKAN \& REICHARD, 1993).

Considerações ambientais e econômicas exigem que os agricultores utilizem menor quantidade de agroquímico pulverizado, procurando melhorar a uniformidade de aplicação.

Estudos apontam que o movimento de rolagem do veículo é responsável por variações na distribuição de volume de 0 a $1.000 \%$, sendo que uma variação de até $100 \%$ seria usual em função das variações de campo (RAMON et al., 1997).

Este trabalho concentra-se no movimento de rolagem da barra do pulverizador, cujo efeito é mostrado na Figura 1. A fim de reduzir a irregularidade do depósito, os pulverizadores agrícolas atuais são equipados com sistema de suspensão a fim de atenuar o movimento de rolagem da barra do pulverizador. A suspensão tem a finalidade de manter o ângulo da barra paralela ao solo, isolando as vibrações do trator que são provocadas pelas irregularidades do solo.

Quase todos os fabricantes têm a sua própria suspensão passiva de barra, sendo mais comumente utilizados dois tipos: pêndulo e trapézio. Os modelos matemáticos para esses dois tipos de suspensão foram discutidos em várias publicações: NATION (1987), O'SULLIVAN (1986), O'SULLIVAN (1998) e FROST \& O'SULLIVAN (1998).

Vários estudos mostram que a utilização de suspensão de barras faz com que a variação da distribuição de volume reduza drasticamente conforme mostrado por LARDOUX et al. (2007a), LARDOUX et al. (2007b), ANTHONIS et al. (2005).

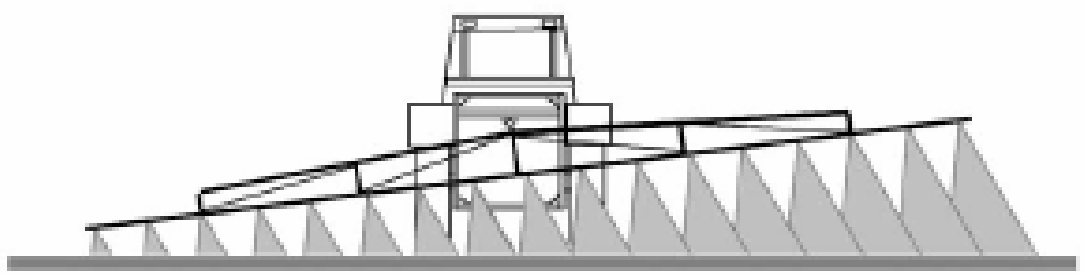

FIGURA 1. Efeito do movimento de rolagem de um pulverizador de barra. Effect of the scroll movement in the spray boom.

O objetivo deste trabalho é a análise da simulação do comportamento dinâmico da barra para duas configurações de suspensão passiva de pulverizadores agrícolas submetidos a pistas de prova padronizadas.

\section{MATERIAL E MÉTODOS}

Para a realização deste trabalho, foram utilizados os modelos matemáticos das duas suspensões mais utilizadas. Construiu-se, a partir do software Matlab ${ }^{\circledR}$, uma rotina para solucionar as equações dos modelos matemáticos empregados. Para estes modelos, também foram feitos os diagramas de blocos com o uso do software Simulink ${ }^{\circledR}$. Por último, estas suspensões foram modeladas pelo método de corpos rígidos, utilizando o software Visual Nastran ${ }^{\circledR}$. Os modelos obtidos através destes três procedimentos são muito próximos (PONTELLI, 2007). Optou-se neste trabalho pela utilização apenas do software Visual Nastran ${ }^{\circledR}$. 


\section{A. Suspensão pendular simples}

A barra está presa no quadro do pulverizador pelo braço $S 3 B 3$. Os pontos $S 3$ e $B 3$ são juntas de revolução que estão ligadas no quadro e na barra, respectivamente. O centro de massa da barra está em $Q$. O veículo é considerado rígido e pode girar sobre um eixo que passa pelo ponto $O$, perpendicular ao plano da Figura 2, representando o eixo de rotação instantâneo do movimento de rolagem do trator. No caso de um trator sem suspensão, o movimento de rolagem é causado pela rotação do eixo traseiro quando uma roda se levanta mais que a outra.

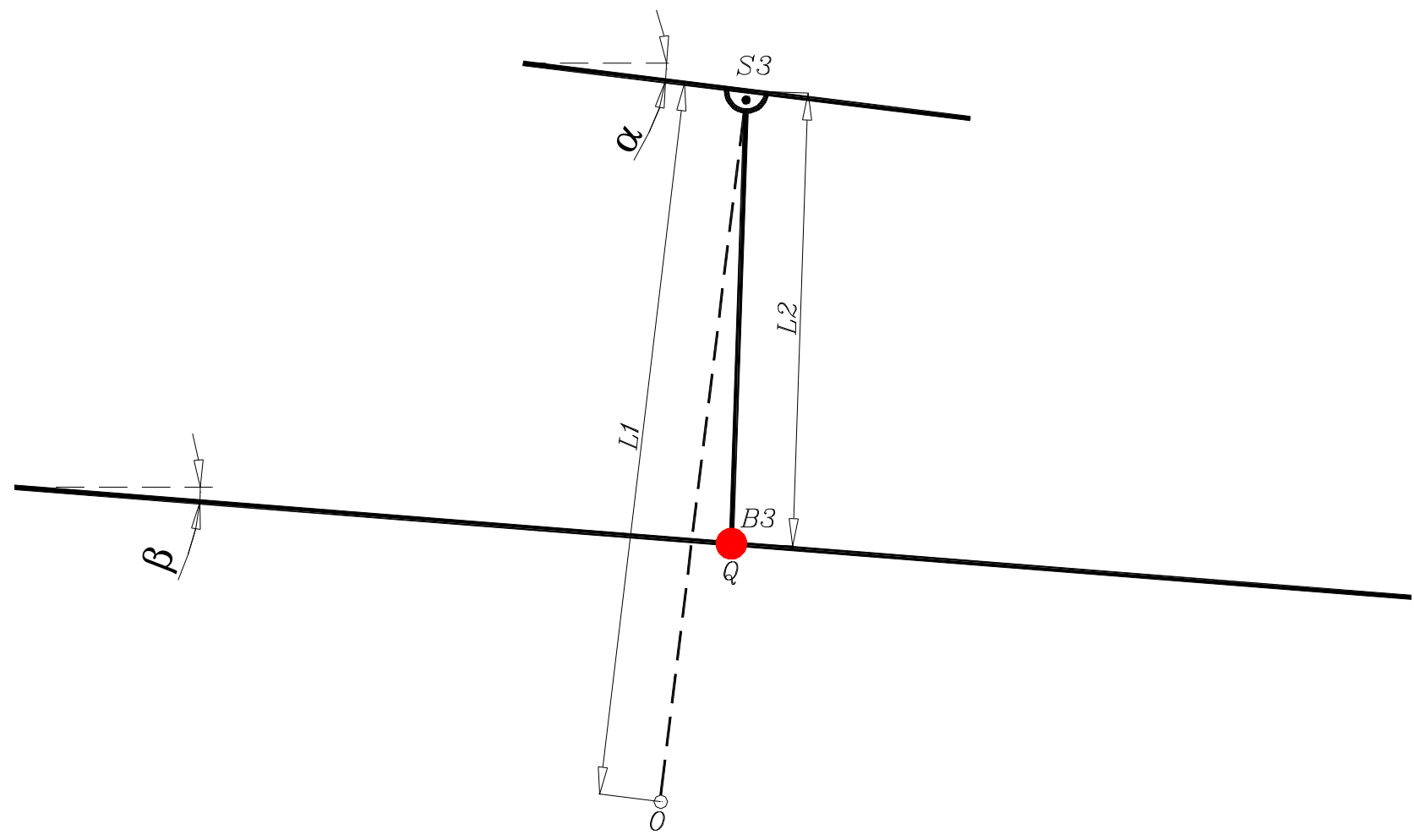

FIGURA 2. Esquema de uma suspensão passiva pendular. Scheme of passive pendulum suspension.

O modelo matemático, que representa a relação entre o ângulo da barra de pulverização $(\beta)$ e o ângulo do quadro do pulverizador $(\alpha)$, foi estudado por O'SULLIVAN (1986). Para pequenos ângulos de rotação, a seguinte função de transferência foi por ele apresentada como:

$$
\frac{\beta}{\alpha}(s)=\frac{E s^{2}+\mu_{R} s}{F s^{2}+\mu_{R} s+G}
$$

em que,

$$
\begin{aligned}
& \mathrm{E}=\mathrm{M}_{\mathrm{B}} \mathrm{L}_{2} \mathrm{~L}_{1} \\
& \mathrm{~F}=\mathrm{I}_{\mathrm{B}}+\mathrm{M}_{\mathrm{B}} \mathrm{L}_{1}^{2} \\
& \mathrm{G}=\mathrm{M}_{\mathrm{B}} \mathrm{gL}_{1}
\end{aligned}
$$

em que,

$$
\frac{\beta}{\alpha}(s) \text { - função resposta em frequência relacionando } \alpha \text { e } \beta \text {; }
$$

$\mathrm{s}$ - operador Laplaciano;

$\mathrm{I}_{\mathrm{B}}$ - momento de inércia da barra sobre o eixo Q perpendicular ao plano da Figura 2, $\mathrm{kg} \mathrm{m}^{2}$;

$\mathrm{L}_{1-2}$ - comprimentos mostrados na Figura 2, m;

$\mathrm{M}_{\mathrm{B}}$ - massa da barra, kg;

$\alpha$ - ângulo de inclinação do trator em relação à horizontal, rad; 
$\beta$ - ângulo de inclinação da barra em relação à horizontal, rad;

$\mu_{R}$ - coeficiente de amortecimento rotacional, $\mathrm{N} \mathrm{m} \mathrm{s/rad,} \mathrm{e}$

$\mathrm{g}$ - aceleração da gravidade, $\mathrm{m} \mathrm{s}^{-2}$.

\section{B. Suspensão trapezoidal - TWIN-LINK}

A barra de pulverização está presa no quadro do pulverizador pelos braços $S 1 B 1$ e $S 2 B 2$. Os pontos $S 1, S 2, B 1$ e $B 2$ são juntas de revolução que estão presas no quadro e na barra, respectivamente. $\mathrm{O}$ centro de massa da barra está em $Q$. O veículo é considerado rígido e pode girar sobre um eixo que passa pelo ponto $O$, perpendicular ao plano da Figura 3, representando o eixo de rotação instantâneo do movimento de rolagem do trator. No caso de um trator sem suspensão, o movimento de rolagem é causado pela rotação do eixo traseiro quando uma roda se levanta mais que a outra.

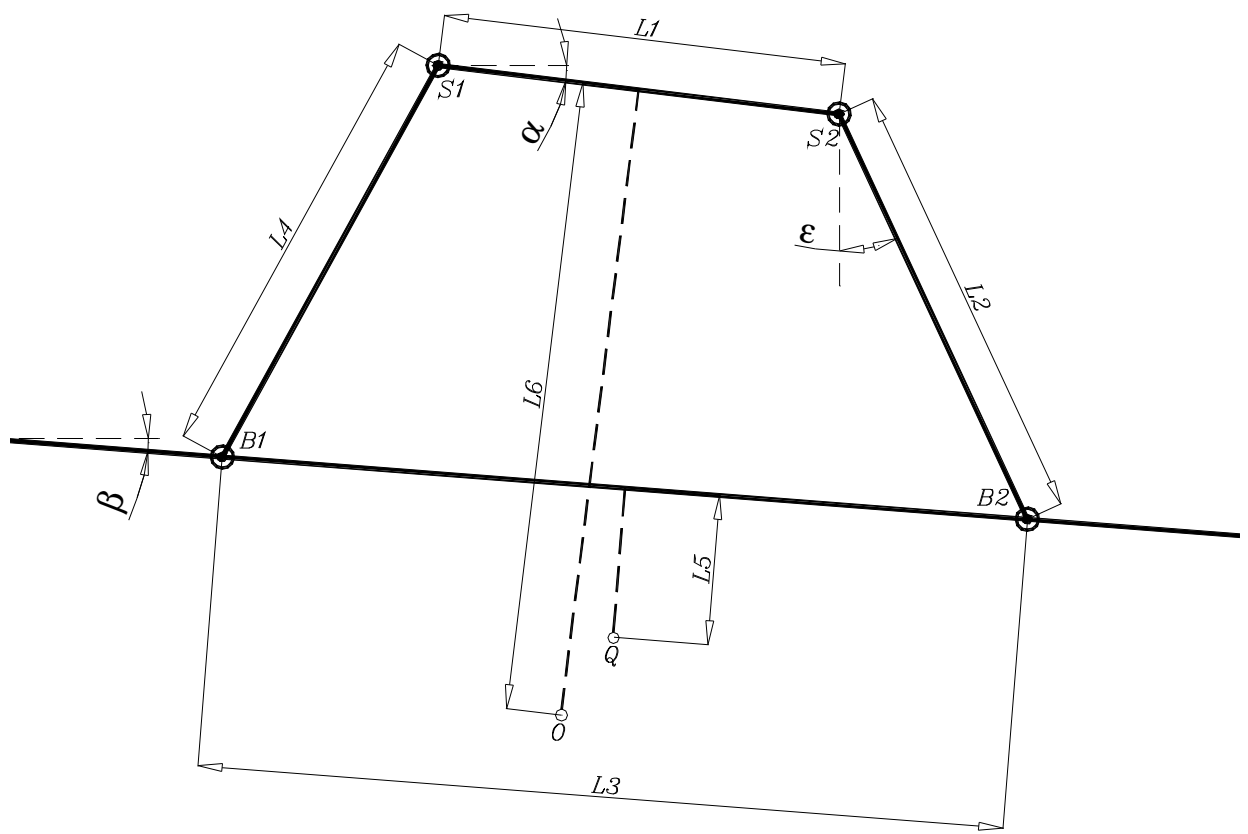

FIGURA 3. Esquema de uma suspensão passiva trapezoidal. Scheme of passive twin-link suspension.

O modelo matemático que representa a relação entre o ângulo da barra de pulverização $(\beta)$ e o ângulo do quadro do pulverizador $(\alpha)$, foi estudado por NATION (1987). Para pequenos ângulos de rotação, a seguinte função de transferência foi por ele apresentada como:

$$
\frac{\beta}{\alpha}(s)=\frac{A s^{2}+\mu_{R} s+B}{C s^{2}+\mu_{R} s+D}
$$

em que,

$$
\begin{aligned}
& A=M_{B}\left[L_{6}+\frac{L_{3}}{2 \tan \bar{\varepsilon}}-L_{2} \cos \bar{\varepsilon}\right]\left[L_{5}+\frac{L_{3}}{2 \tan \bar{\varepsilon}}\right] \\
& B=M_{B} g\left[\frac{\left(L_{3} / 2\right)\left(\left(L_{3} / 2\right)-L_{2} \operatorname{sen}^{3} \bar{\varepsilon}\right)}{L_{2} \cos \bar{\varepsilon} \operatorname{sen}^{2} \bar{\varepsilon}}-\frac{L_{3}}{2 \tan \bar{\varepsilon}}\right] \\
& C=I_{B}+M_{B}\left[L_{5}+\frac{L_{3}}{2 \tan \bar{\varepsilon}}\right]^{2}
\end{aligned}
$$




$$
\begin{aligned}
& \mathrm{D}=\mathrm{M}_{\mathrm{B}}\left[\mathrm{L}_{5}+\frac{\left(\mathrm{L}_{3} / 2\right)\left(\left(\mathrm{L}_{3} / 2\right)-\mathrm{L}_{2} \operatorname{sen}^{3} \bar{\varepsilon}\right)}{\mathrm{L}_{3} \cos \bar{\varepsilon} \operatorname{sen}^{2} \bar{\varepsilon}}\right] \\
& \bar{\varepsilon}=\operatorname{sen}^{-1}\left[\frac{\left(\mathrm{L}_{3}-\mathrm{L}_{1}\right)}{\mathrm{L}_{2}}\right]
\end{aligned}
$$

em que,

$\frac{\beta}{\alpha}(s)$ - função resposta em frequência relacionando $\alpha$ e $\beta$;

$\mathrm{s}$ - operador Laplaciano;

$\mathrm{I}_{\mathrm{B}}$ - momento de inércia da barra sobre o eixo Q perpendicular ao plano da Figura $3, \mathrm{~kg} \mathrm{~m}^{2}$;

$\mathrm{L}_{1-6}$ - comprimentos mostrados na Figura 3, m;

$\mathrm{M}_{\mathrm{B}}$ - massa da barra, kg;

$\alpha$ - ângulo de inclinação do trator em relação à horizontal, rad;

$\beta$ - ângulo de inclinação da barra em relação à horizontal, rad;

$\bar{\varepsilon}$ - ângulo médio quando $\alpha=0$ e $\beta=0$, rad;

$\mu_{R}$ - coeficiente de amortecimento rotacional, $\mathrm{N} \mathrm{m} \mathrm{s/rad,} \mathrm{e}$

$\mathrm{g}$ - aceleração da gravidade, $\mathrm{m} / \mathrm{s}^{2}$.

Para realizar as simulações, foram utilizados os seguintes parâmetros referentes a um modelo virtual em desenvolvimento que possui uma barra de pulverização de $50 \mathrm{~m}$ de comprimento:

Massa da Barra $=732 \mathrm{~kg}$;

Momento de Inércia $=98.000 \mathrm{~kg} \cdot \mathrm{m}^{2}$;

Pendulo Simples: $\mathrm{L} 1=0,7 \mathrm{~m}, \mathrm{~L} 2=0,2 \mathrm{~m}, \mu_{\mathrm{r}}=18.985 \mathrm{~N} \mathrm{~m} \mathrm{~s} / \mathrm{rad}$., e

Trapezoidal : $\mathrm{L} 1=1,0 \mathrm{~m}, \mathrm{~L} 2=\mathrm{L} 4=0,30 \mathrm{~m}, \mathrm{~L} 3=1,50 \mathrm{~m}, \mathrm{~L} 6=0,565 \mathrm{~m}$

$\varepsilon=57^{\circ}, \mu_{\mathrm{r}}=82312 \mathrm{~N} \mathrm{~m} \mathrm{~s} / \mathrm{rad}$.

Estes valores foram obtidos através do software de modelamento em 3D Pro-Engineer.

\section{RESULTADOS E DISCUSSÃO}

Foram analisados dois tipos de suspensão em condições estipuladas pela norma de vibração (ISO 5008, 2002). Nesta norma, estão descritos dois tipos de pista de prova: pista de prova acidentada e pista de prova suavizada. Também como parâmetros de avaliação foram utilizadas duas velocidades de translado do pulverizador: $5 \mathrm{~km} \mathrm{~h}^{-1}$ e $15 \mathrm{~km} \mathrm{~h}^{-1}$.

Em todas as análises simuladas, foram obtidos os valores do ângulo de inclinação do trator ( $\alpha$ ) a partir de dados de entrada correspondentes ao deslocamento vertical virtual de cada tipo de pista. Os valores do ângulo da barra $(\beta)$ foram obtidos a partir dos resultados das simulações de cada tipo de mecanismo de suspensão, pendular e trapezoidal.

Em uma primeira análise, foram obtidos os valores para a média aritmética, máximo, mínimo e desvio-padrão das variáveis de entrada e de saída, com o objetivo de se verificar de uma maneira geral se as suspensões estariam atenuando os valores de entrada (ângulo do trator).

Em uma segunda etapa, foram feitas análises da resposta em frequência, a partir do sinal temporal das variáveis simuladas. Foi utilizada a transformada rápida de Fourier, FFT, com duas frequências de amostragem distintas para se observarem os fenômenos que podem ocorrer em baixa frequência (amostragem de $2 \mathrm{~Hz}$ ) e alta frequência (amostragem de $100 \mathrm{~Hz}$ ).

Quando foi utilizada a frequência de amostragem de $2 \mathrm{~Hz}$, foi utilizada uma decimação (dados tomados a cada intervalo previamente escolhido) de $128 \mathrm{~Hz}$ e, quando foi utilizada a frequência de 
amostragem de $100 \mathrm{~Hz}$, foi utilizada uma decimação de $512 \mathrm{~Hz}$. Essa estratégia foi adotada com o objetivo de não perder definição do sinal nem deixá-lo com muito ruído, atendendo aos requisitos do teorema da amostragem (DOEBELIN, 1985).

A escolha das faixas de frequências para realizar as análises dos dados simulados foi feita utilizando como critérios as frequências naturais usuais nestes equipamentos $(0,2 \mathrm{a} 0,3 \mathrm{~Hz})$. Assim, consideram-se aqui baixas frequências valores inferiores a $0,2 \mathrm{~Hz}$. Na prática, tais frequências ocorrem quando o equipamento transpõe terraços em condições de campo. A outra faixa, acima de $2 \mathrm{~Hz}$, está relacionada às condições de cultura encontradas em campo, ou seja, pequenos obstáculos, próximos um do outro, provocando altas frequências de excitação do trator. Frequências na faixa intermediária de 0,2 a 2 Hz não são encontradas nas aplicações práticas destes equipamentos.

\section{Pista suavizada $\left(5 \mathrm{~km} \mathrm{~h}^{-1}\right)$}

$\mathrm{Na}$ Figura 4, mostra-se o comportamento das suspensões passivas para barras de pulverizadores quando são submetidas a uma entrada da norma ISO 5008 (2002), correspondente a uma pista de prova suavizada, com velocidade de deslocamento de $5 \mathrm{~km} \mathrm{~h}^{-1}$.

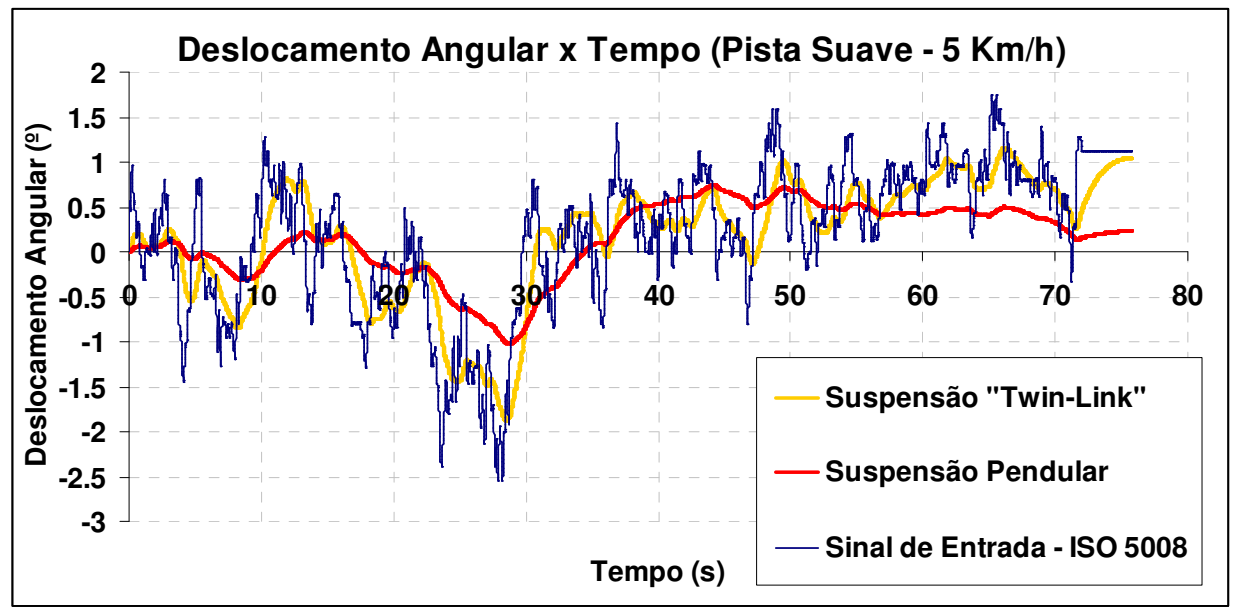

FIGURA 4. Comportamento das suspensões para uma entrada de pista de prova suavizada, com velocidade de deslocamento de $5 \mathrm{~km} \mathrm{~h}^{-1}$. Suspensions behavior for a smooth track race, with the forward speed of $5 \mathrm{~km} \mathrm{~h}^{-1}$.

Na Tabela 1, mostram-se os dados do sinal das suspensões quando submetidas a um sinal de pista de prova suave (ISO 5008, 2002), com velocidade de deslocamento de $5 \mathrm{~km} \mathrm{~h}^{-1}$.

TABELA 1. Dados dos sinais das suspensões submetidas a uma entrada de pista suavizada com velocidade de translado de $5 \mathrm{~km} \mathrm{~h}^{-1}$. Data from the suspensions signals submitted to a smooth track race with a transfer speed of $5 \mathbf{~ k m ~ h}^{-1}$.

\begin{tabular}{lccc}
\hline (graus) & Sinal de Entrada $(\alpha)$ & Suspensão Pendular $(\beta)$ & Suspensão Trapezoidal $(\beta)$ \\
\hline Média & 0,20 & 0,14 & 0,18 \\
Máximo & 1,75 & 0,74 & 1,16 \\
Mínimo & $-2,55$ & $-1,03$ & $-1,88$ \\
Desvio-Padrão & 0,80 & 0,42 & 0,67 \\
\hline
\end{tabular}

Na Figura 5, mostra o comportamento das suspensões passivas para barras de pulverizadores quando submetidas a uma análise através de FFT com uma frequência de amostragem de $100 \mathrm{~Hz}$, com objetivo de avaliar as repostas das suspensões em alta frequência $(\omega>2 \mathrm{~Hz})$. Esta análise foi feita na pista de prova normalizada ISO 5008 (2002) suavizada, com velocidade de translado do equipamento de $5 \mathrm{~km} \mathrm{~h}^{-1}$. 


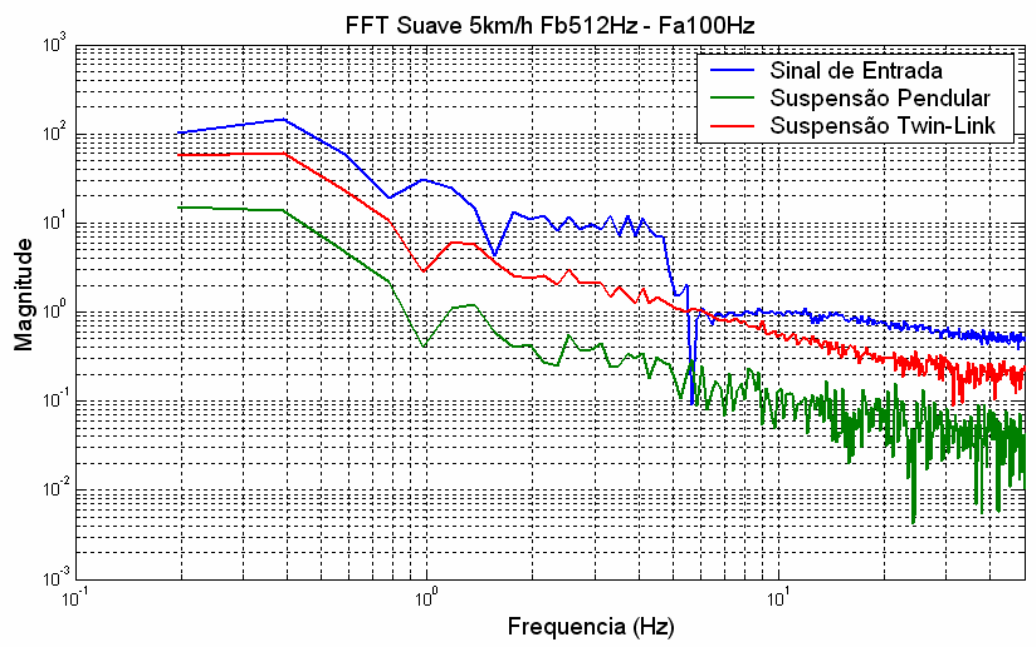

FIGURA 5. Análise da FFT com frequência de amostragem de $100 \mathrm{~Hz}$ - Pista de Prova ISO 5008 (2002) suavizada, com velocidade de $5 \mathrm{~km} \mathrm{~h}^{-1}$. Analysis of the FFT with a sampling frequency of $100 \mathrm{~Hz}$ - Track Test ISO 5008 smoothed with a speed of $5 \mathrm{~km} \mathrm{~h}^{-1}$.

É possível observar, pela análise da Figura 5, que a suspensão pendular absorve mais as entradas em toda a extensão da faixa de frequência analisada. Portanto, pode-se concluir que a suspensão pendular é mais eficiente que a suspensão trapezoidal na faixa de frequência analisada $(\omega>2 \mathrm{~Hz})$.

$\mathrm{Na}$ Figura 6, apresenta-se o comportamento das suspensões passivas para barras de pulverizadores quando submetidas a uma análise de FFT, com uma frequência de amostragem de $2 \mathrm{~Hz}$, com o objetivo de avaliar as repostas das suspensões em baixas frequências $(\omega<0,2 \mathrm{~Hz})$. Esta análise foi feita nas condições de pista de prova normalizada ISO 5008 (2002) suavizada, com velocidade de translado do equipamento de $5 \mathrm{~km} \mathrm{~h}^{-1}$.

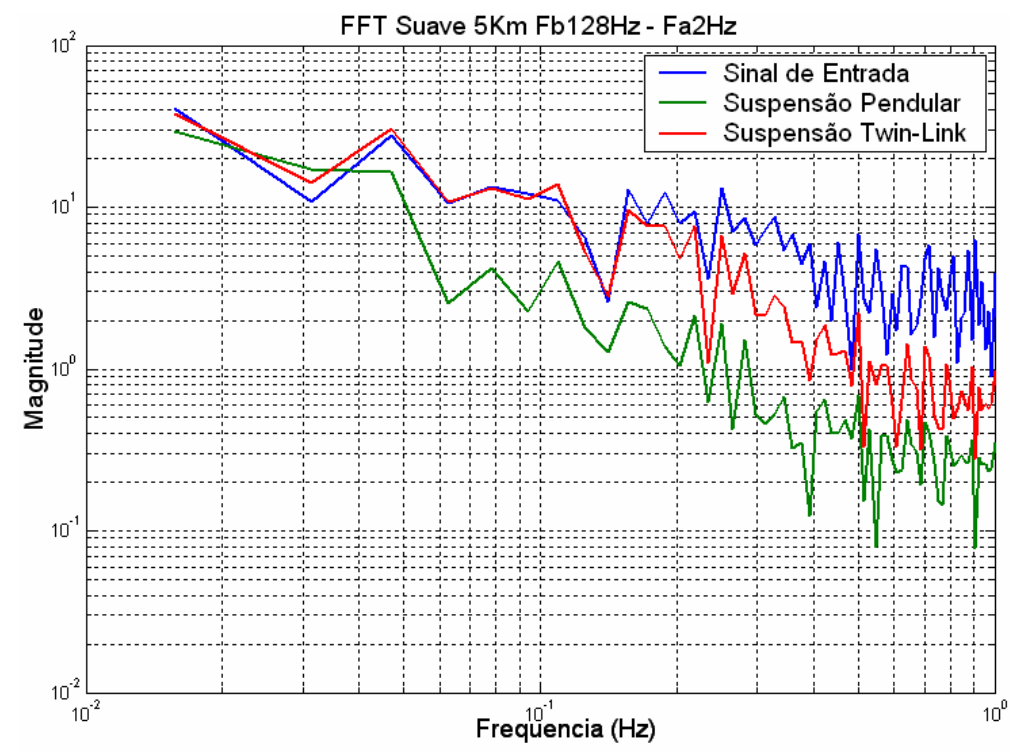

FIGURA 6. Análise por FFT com frequência de amostragem de $2 \mathrm{~Hz}$ - Pista de Prova ISO 5008 (2002) suavizada, com velocidade de $5 \mathrm{~km} \mathrm{~h}^{-1}$. Analysis of the FFT with a sampling frequency of $2 \mathrm{~Hz}$ - Track Test ISO 5008 smoothed with a speed of $5 \mathrm{~km} \mathrm{~h}^{-1}$.

É possível observar, pela análise da Figura 6, que a suspensão Twin-Link acompanha o sinal de entrada até a frequência de $0,2 \mathrm{~Hz}$. Isto significa que esta suspensão satisfaz o critério de acompanhar a entrada em baixas frequências. Nessa situação, a barra do pulverizador consegue 
ficar paralela ao quadro do pulverizador e isso corresponde a melhor qualidade de aplicação de defensivo (RAMON et al., 1997). Já a suspensão pendular não consegue acompanhar o quadro do pulverizador, causando um erro durante a aplicação.

\section{Pista suavizada (15 $\left.\mathrm{km} \mathrm{h}^{-1}\right)$}

$\mathrm{Na}$ Figura 7, apresenta-se o comportamento das suspensões passivas para barras de pulverizadores quando são submetidas a uma entrada da norma ISO 5008 (2002) do tipo pista de prova suavizada, com velocidade de deslocamento de $15 \mathrm{~km} \mathrm{~h}^{-1}$.

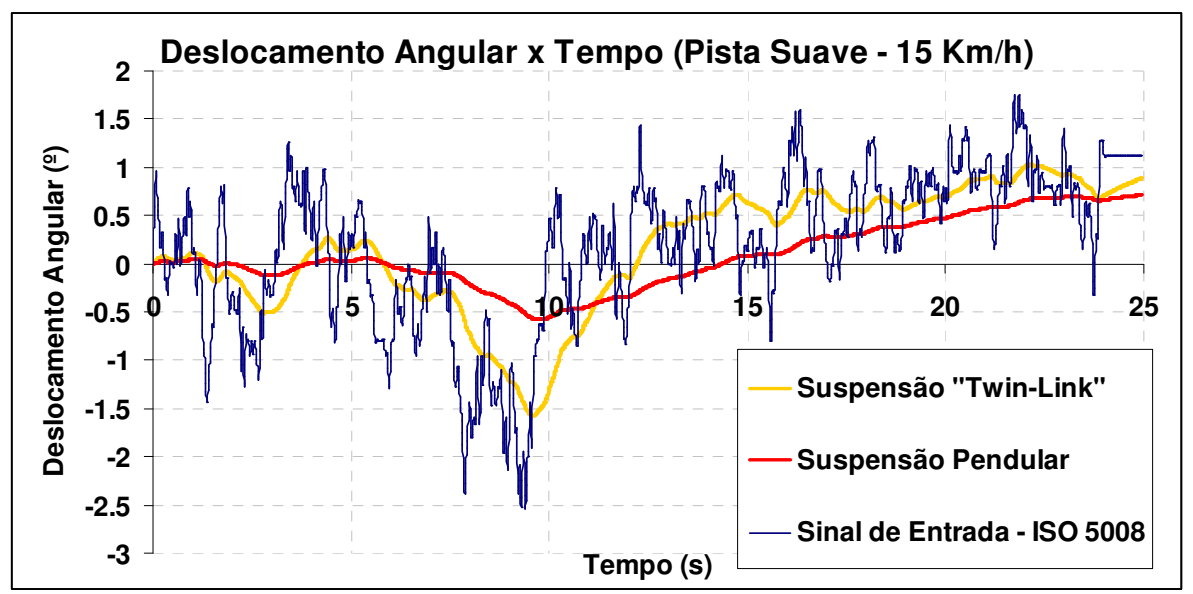

FIGURA 7. Comportamento das suspensões para uma entrada de pista de prova suave, com velocidade de deslocamento de $15 \mathrm{~km} \mathrm{~h}^{-1}$. Suspensions behavior for a smooth track race, with the forward speed of $15 \mathrm{~km} \mathrm{~h}^{-1}$.

Na Tabela 2, mostram-se os dados do sinal das suspensões quando submetidas a um sinal de pista de prova suave (ISO 5008, 2002), com velocidade de deslocamento de $15 \mathrm{~km} \mathrm{~h}^{-1}$.

TABELA 2. Dados dos sinais das suspensões submetidas a uma entrada de pista suavizada, com velocidade de translado de $15 \mathrm{~km} \mathrm{~h}^{-1}$. Data from the suspensions signals submitted to a smooth track race with a transfer speed of $15 \mathrm{~km} \mathrm{~h}^{-1}$.

\begin{tabular}{cccc}
\hline (graus) & Sinal de Entrada $(\alpha)$ & Suspensão Pendular $(\beta)$ & Suspensão Trapezoidal $(\beta)$ \\
\hline Média & 0,19 & 0,09 & 0,17 \\
Máximo & 1,75 & 0,71 & 1,03 \\
Mínimo & $-2,54$ & $-0,57$ & $-1,58$ \\
Desvio-Padrão & 0,80 & 0,36 & 0,63 \\
\hline
\end{tabular}

$\mathrm{Na}$ Figura 8, mostra-se o comportamento das suspensões passivas para barras de pulverizadores quando submetidas a uma análise de FFT, com uma frequência de amostragem de $100 \mathrm{~Hz}$, com objetivo de avaliar as repostas das suspensões em alta frequência $(\omega>2 \mathrm{~Hz})$. Esta análise foi feita na pista de prova normalizada ISO 5008 (2002) suavizada, com velocidade de translado do equipamento de $15 \mathrm{~km} \mathrm{~h}^{-1}$.

É possível observar, pela análise da Figura 8, que a suspensão pendular absorve mais as entradas em toda a extensão da faixa de frequência analisada. Portanto, pode-se concluir que a suspensão pendular é mais eficiente que a suspensão trapezoidal na faixa de frequência analisada $(\omega>2 \mathrm{~Hz})$. 


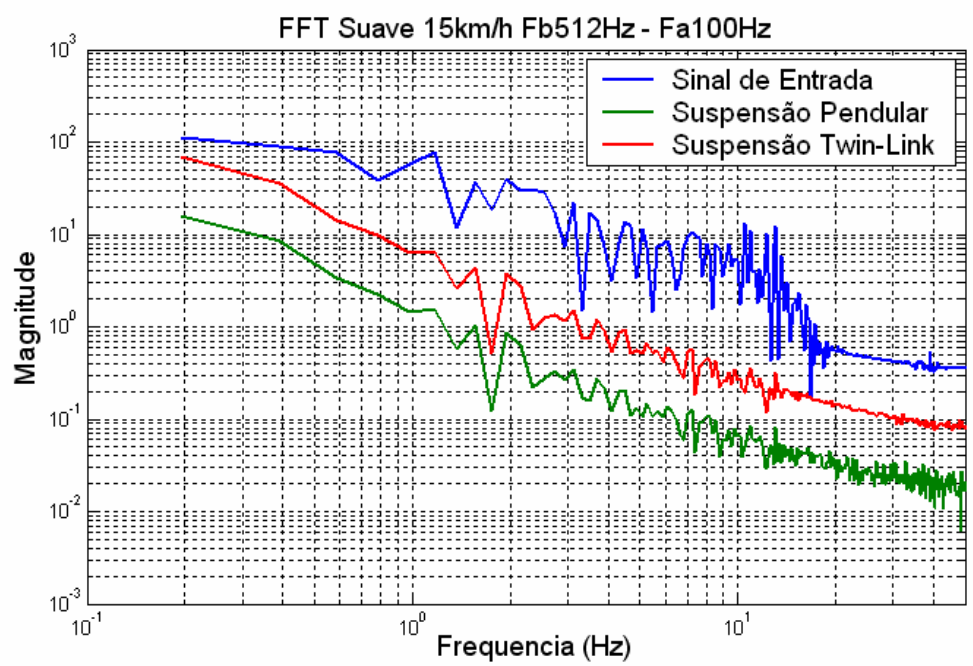

FIGURA 8. Análise da FFT com frequência de amostragem de $100 \mathrm{~Hz}$ - Pista de Prova ISO 5008 (2002) suavizada, com velocidade de $15 \mathrm{~km} \mathrm{~h}^{-1}$. Analysis of the FFT with a sampling frequency of $100 \mathrm{~Hz}$ - Track Test ISO 5008 smoothed with a speed of $15 \mathrm{~km} \mathrm{~h}^{-1}$.

Na Figura 9, apresenta-se o comportamento das suspensões passivas para barras de pulverizadores quando submetidas a uma análise de FFT, com uma frequência de amostragem de $2 \mathrm{~Hz}$, com objetivo de avaliar as repostas das suspensões em baixas frequências $(\omega<0,2 \mathrm{~Hz})$. Esta análise foi feita na pista de prova normalizada ISO 5008 (2002) suavizada, com velocidade de translado do equipamento de $15 \mathrm{~km} \mathrm{~h}^{-1}$.

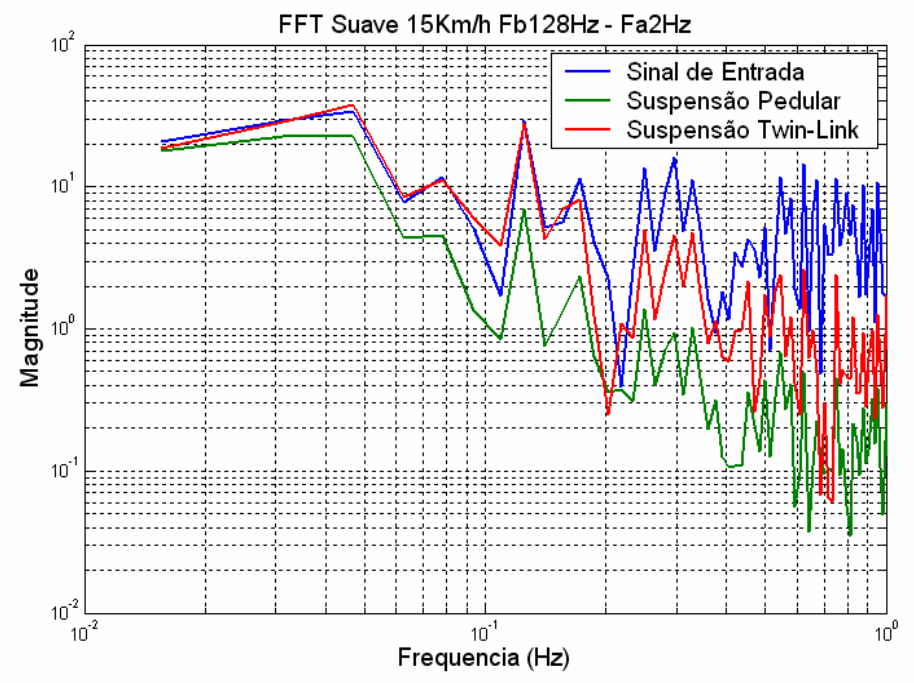

FIGURA 9. Análise por FFT com frequência de amostragem de $2 \mathrm{~Hz}$ - Pista de Prova ISO 5008 (2002) suavizada, com velocidade de $15 \mathrm{~km} \mathrm{~h}^{-1}$. Analysis of the FFT with a sampling frequency of $2 \mathrm{~Hz}$ - Track Test ISO 5008 smoothed with a speed of $15 \mathrm{~km} \mathrm{~h}^{-1}$.

É possível observar, pela análise da Figura 9, que a suspensão Twin-Link acompanha o sinal de entrada até a frequência de $0,2 \mathrm{~Hz}$. Isto significa que esta suspensão satisfaz o critério de acompanhar a entrada em baixas frequências. Nessa situação, a barra do pulverizador consegue ficar paralela ao quadro do pulverizador. Isso corresponde a melhor qualidade de aplicação de defensivo (RAMON et al., 1997). Já a suspensão pendular não consegue acompanhar o quadro do pulverizador nestas condições, causando um erro durante a aplicação. 


\section{Pista acidentada $\left(5 \mathrm{~km} \mathrm{~h}^{-1}\right)$}

Na Figura 10, apresenta-se o comportamento das suspensões passivas para barras de pulverizadores quando são submetidas a uma entrada da norma ISO 5008 (2002) de uma pista de prova acidentada, com velocidade de deslocamento de $5 \mathrm{~km} \mathrm{~h}^{-1}$.

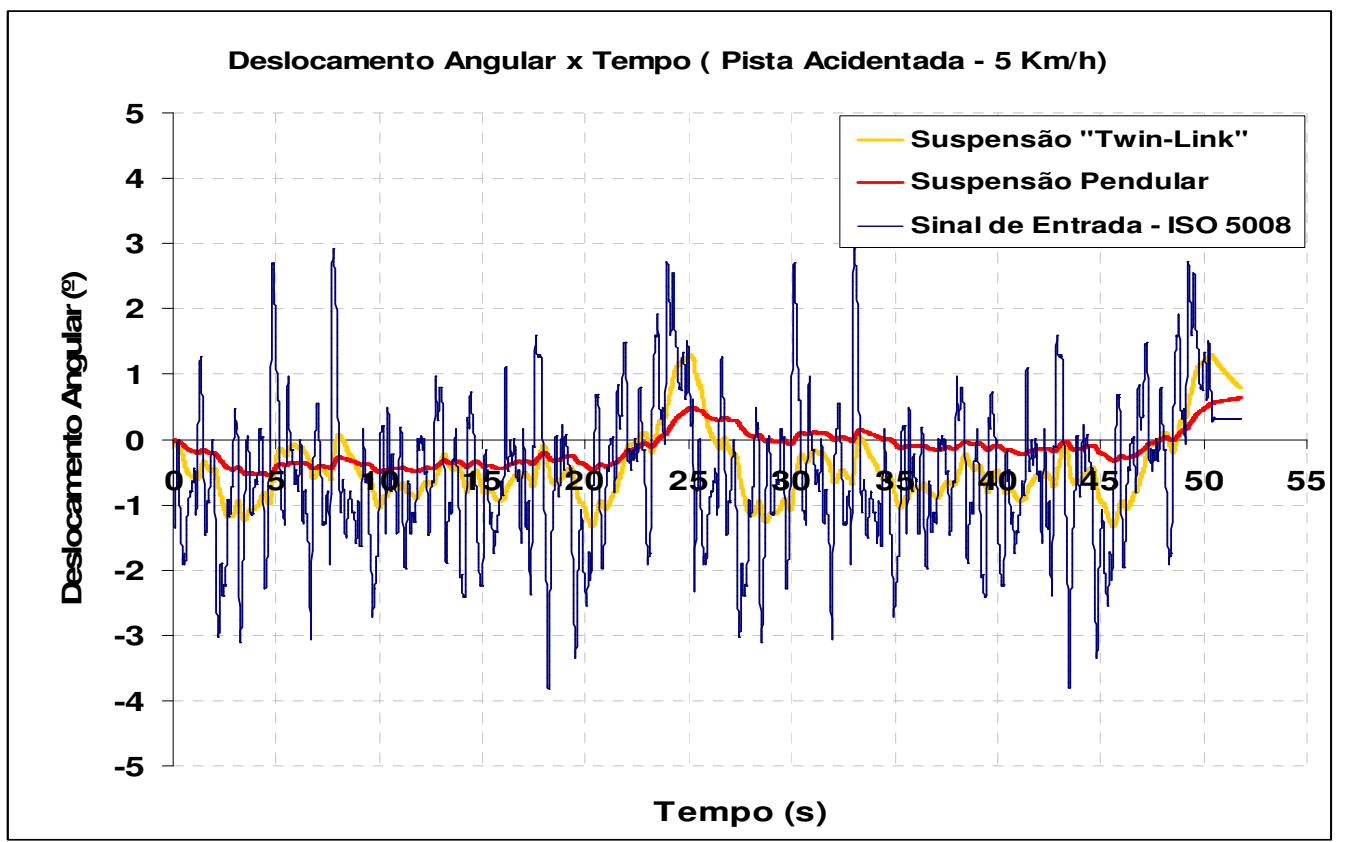

FIGURA 10. Comportamento das suspensões para uma entrada de pista de prova acidentada, com velocidade de deslocamento $5 \mathrm{~km} \mathrm{~h}^{-1}$. Suspensions behavior for a bumpy track race, with the forward speed of $5 \mathrm{~km} \mathrm{~h}^{-1}$.

Na Tabela 3, mostram-se os dados do sinal das suspensões quando submetidas a um sinal de pista de prova acidentada (ISO 5008, 2002), com velocidade de deslocamento de $5 \mathrm{~km} \mathrm{~h}^{-1}$.

TABELA 3. Dados dos sinais das suspensões submetidas a uma entrada de pista acidentada, com velocidade de translado de $5 \mathrm{~km} \mathrm{~h}^{-1}$. Data from the suspensions signals submitted to a bumpy track race with a transfer speed of $5 \mathrm{~km} \mathrm{~h}^{-1}$.

\begin{tabular}{cccc}
\hline (graus) & Sinal de Entrada $(\alpha)$ & Suspensão Pendular $(\beta)$ & Suspensão Trapezoidal $(\beta)$ \\
\hline Média & $-0,53$ & $-0,14$ & $-0,43$ \\
Máximo & 2,93 & 0,63 & 1,29 \\
Mínimo & $-3,83$ & $-0,56$ & $-1,34$ \\
Desvio-Padrão & 1,12 & 0,27 & 0,57 \\
\hline
\end{tabular}

Na Figura 11, apresenta-se o comportamento das suspensões passivas para barras de pulverizadores quando são submetidas a uma análise de FFT, com uma frequência de amostragem de $100 \mathrm{~Hz}$, com objetivo de avaliar as repostas das suspensões em alta frequência $(\omega>2 \mathrm{~Hz})$. Esta análise foi feita na pista de prova normalizada ISO 5008 (2002) acidentada, com velocidade de translado do equipamento de $5 \mathrm{~km} \mathrm{~h}^{-1}$.

É possível observar, pela análise da Figura 11, que a suspensão pendular absorve mais as entradas até a frequência de $2 \mathrm{~Hz}$. Após esta frequência de excitação, a suspensão trapezoidal passa a ser a mais eficiente, pois absorve melhor as irregularidades da entrada, apresentando menor variação na aplicação de defensivo. 


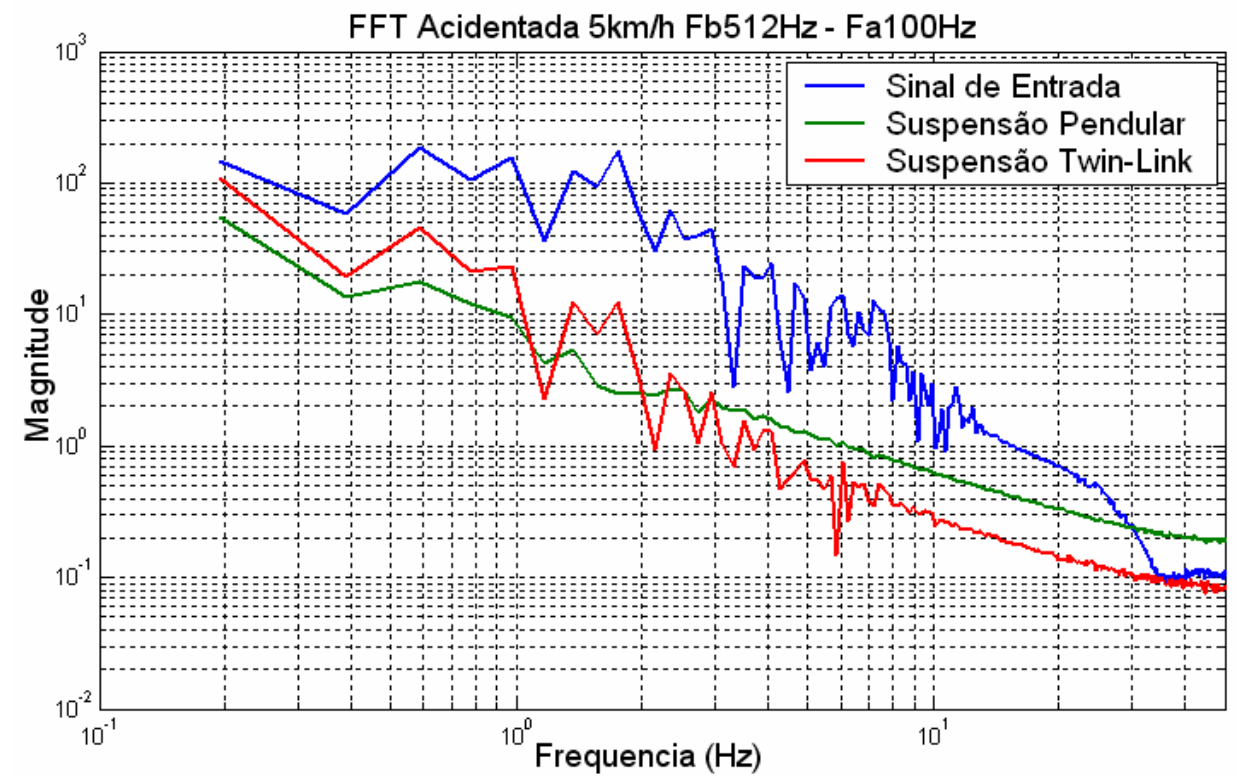

FIGURA 11. Análise da FFT com frequência de amostragem de $100 \mathrm{~Hz}$ - Pista de Prova ISO 5008 (2002) acidentada, com velocidade de $5 \mathrm{~km} \mathrm{~h}^{-1}$. Analysis of the FFT with a sampling frequency of $100 \mathrm{~Hz}$ - Track Test ISO 5008 bumpy with a speed of $5 \mathrm{~km} \mathrm{~h}^{-1}$.

$\mathrm{Na}$ Figura 12, mostra-se o comportamento das suspensões passivas para barras de pulverizadores quando submetidas a uma análise de FFT, com uma frequência de amostragem de $2 \mathrm{~Hz}$, com objetivo de avaliar as repostas das suspensões em baixas frequências $(\omega<0,2 \mathrm{~Hz})$. Esta análise foi feita na pista de prova normalizada ISO 5008 (2002) acidentada, com velocidade de translado do equipamento de $5 \mathrm{~km} \mathrm{~h}^{-1}$.

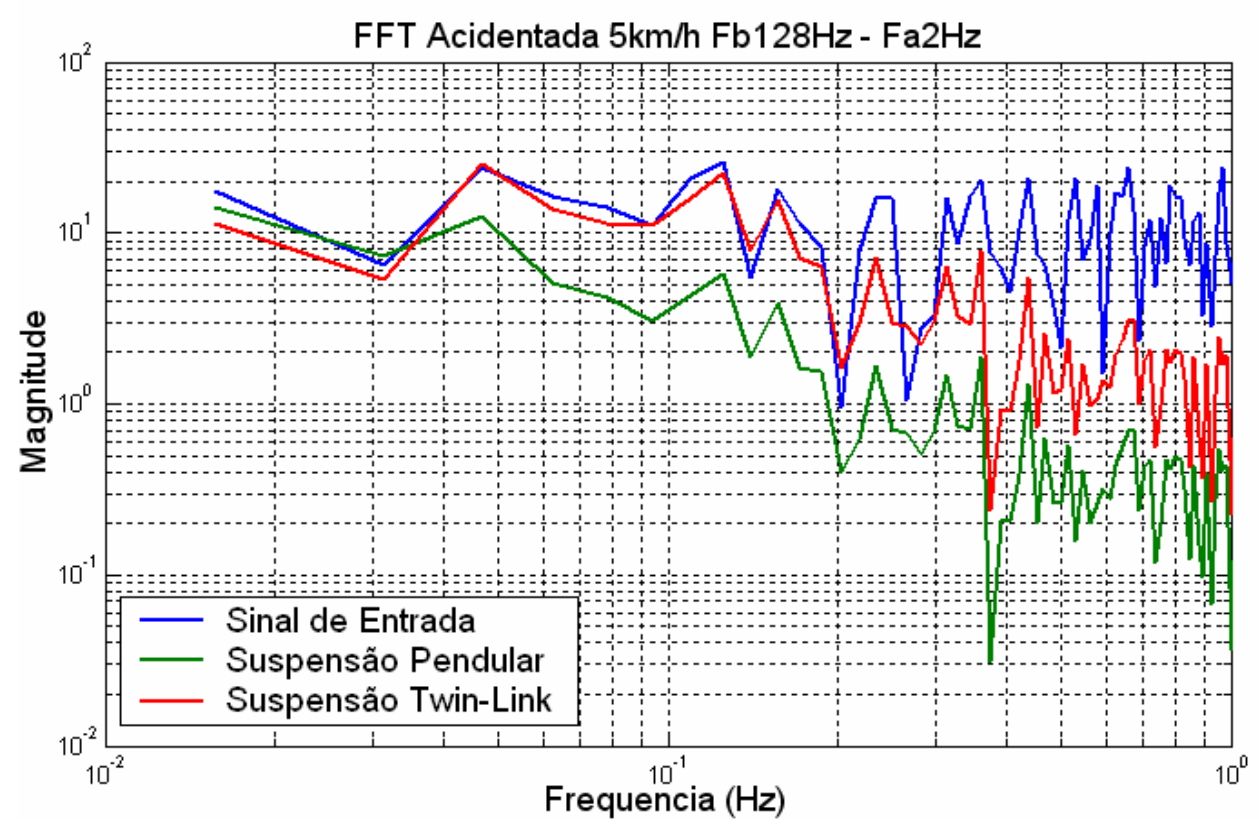

FIGURA 12. Análise da FFT com frequência de amostragem de $2 \mathrm{~Hz}$ - Pista de Prova ISO 5008 (2002) acidentada, com velocidade de $5 \mathrm{~km} \mathrm{~h}^{-1}$. Analysis of the FFT with a sampling frequency of $2 \mathrm{~Hz}$ - Track Test ISO 5008 bumpy with a speed of $5 \mathrm{~km} \mathrm{~h}^{-1}$.

É possível observar, pela análise da Figura 12, que a suspensão Twin-Link acompanha o sinal de entrada até a frequência de 0,2 Hz. Isto significa que esta suspensão satisfaz o critério de 
acompanhar a entrada em baixas frequências. Nessa situação, a barra do pulverizador consegue ficar paralela ao quadro do pulverizador, o que corresponde a melhor qualidade de aplicação de defensivo (RAMON et al., 1997). Já a suspensão pendular não consegue acompanhar o quadro do pulverizador, causando um erro durante a aplicação.

\section{Pista acidentada $\left(15 \mathrm{~km} \mathrm{~h}^{-1}\right)$}

$\mathrm{Na}$ Figura 13, apresenta-se o comportamento das suspensões passivas para barras de pulverizadores quando submetidas a uma entrada da norma ISO 5008 (2002) do tipo pista de prova acidentada, com velocidade de deslocamento de $15 \mathrm{~km} \mathrm{~h}^{-1}$.

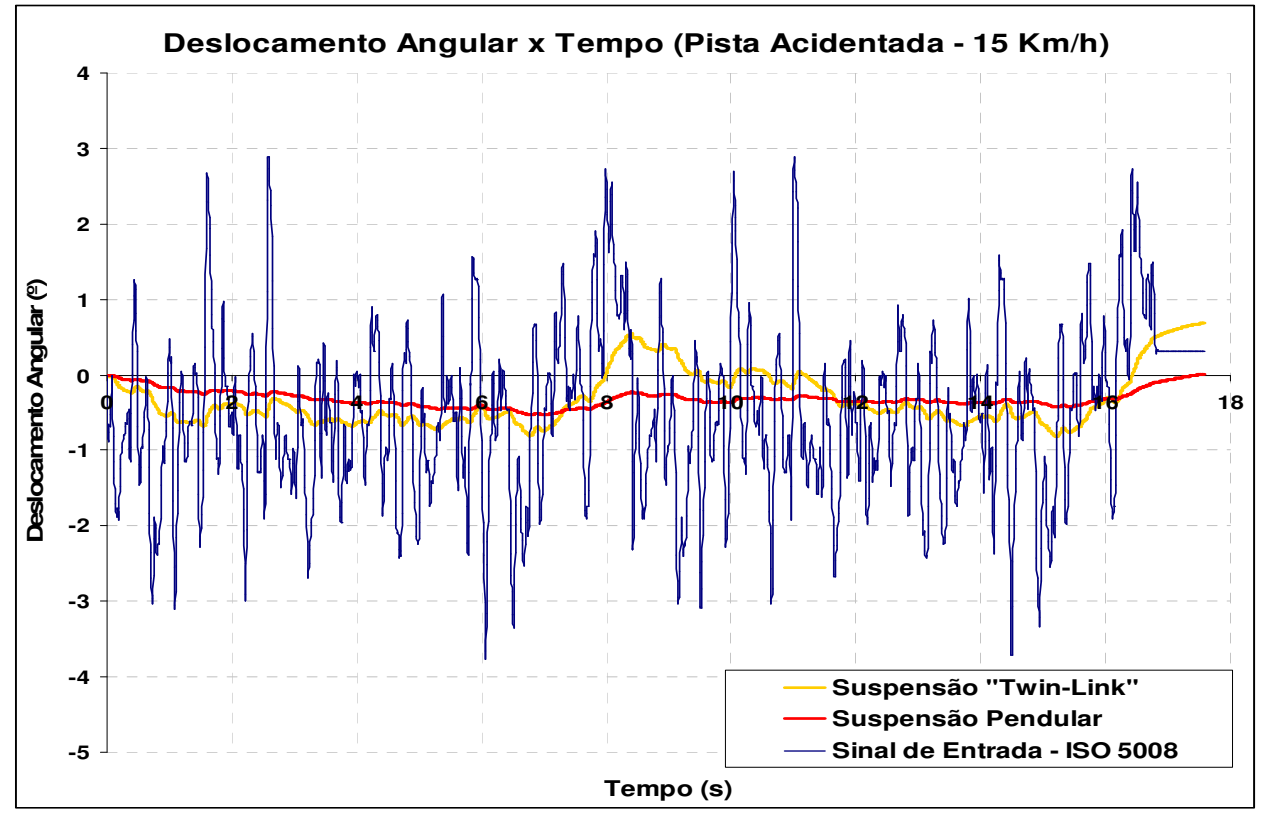

FIGURA 13. Comportamento das suspensões para uma entrada de pista de prova acidentada, com velocidade de deslocamento de $15 \mathrm{~km} \mathrm{~h}^{-1}$. Suspensions behavior for a bumpy track race, with the forward speed of $15 \mathrm{~km} \mathrm{~h}^{-1}$.

Na Tabela 4, apresentam-se os dados do sinal das suspensões quando submetidas a um sinal de pista de prova acidentada (ISO 5008, 2002), com velocidade de deslocamento de $15 \mathrm{~km} \mathrm{~h}^{-1}$.

TABELA 4. Dados dos sinais das suspensões submetidas a uma entrada de pista acidentada, com velocidade de translado de $15 \mathrm{~km} \mathrm{~h}^{-1}$. Data from the suspensions signals submitted to a bumpy track race with a transfer speed of $15 \mathrm{~km} \mathrm{~h}^{-1}$.

\begin{tabular}{lccc}
\hline (graus) & Sinal de Entrada $(\alpha)$ & Suspensão Pendular $(\beta)$ & Suspensão Trapezoidal $(\beta)$ \\
\hline Média & $-0,51$ & $-0,31$ & $-0,31$ \\
Máximo & 2,90 & 0,01 & 0,69 \\
Mínimo & $-3,76$ & $-0,53$ & $-0,82$ \\
Desvio-Padrão & 1,12 & 0,11 & 0,37 \\
\hline
\end{tabular}

$\mathrm{Na}$ Figura 14, mostra-se o comportamento das suspensões passivas para barras de pulverizadores quando submetidas a uma análise de FFT, com uma frequência de amostragem de $100 \mathrm{~Hz}$, com objetivo de avaliar as repostas das suspensões em alta frequência $(\omega>2 \mathrm{~Hz})$. Esta análise foi feita na pista de prova normalizada ISO 5008 (2002) do tipo acidentada, com velocidade de translado do equipamento de $15 \mathrm{~km} \mathrm{~h}^{-1}$. 


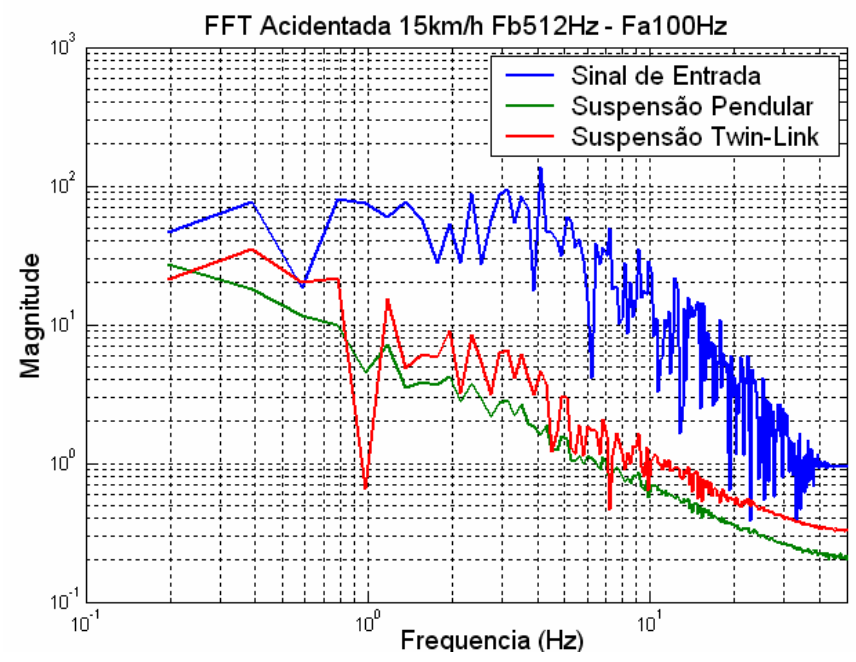

FIGURA 14. Análise da FFT com frequência de amostragem de $100 \mathrm{~Hz}$ - Pista de Prova ISO 5008 (2002) acidentada, com velocidade de $15 \mathrm{~km} \mathrm{~h}^{-1}$. Analysis of the FFT with a sampling frequency of $100 \mathrm{~Hz}$ - Track Test ISO 5008 bumpy with a speed of $15 \mathrm{~km} \mathrm{~h}^{-1}$.

É possível observar, pela análise da Figura 14, que a suspensão pendular absorve mais as entradas em relação à suspensão trapezoidal em toda a extensão da faixa de frequência analisada. Portanto, pode-se concluir que a suspensão pendular é mais eficiente que a suspensão trapezoidal na faixa de frequência analisada.

Na Figura 15, apresenta-se o comportamento das suspensões passivas para barras de pulverizadores quando submetidas à análise de FFT, com uma frequência de amostragem de $2 \mathrm{~Hz}$, com objetivo de avaliar as repostas das suspensões em baixas frequências $(\omega<0,2 \mathrm{~Hz})$. Esta análise foi feita na pista de prova normalizada ISO 5008 (2002) do tipo acidentada, com velocidade de translado do equipamento de $15 \mathrm{~km} \mathrm{~h}^{-1}$.

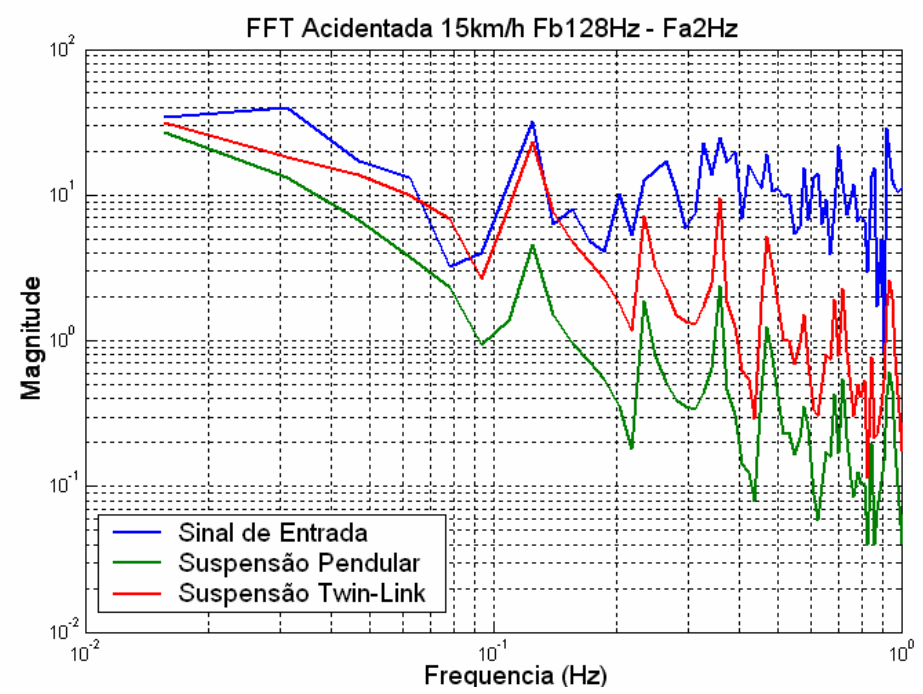

FIGURA 15. Análise da FFT com frequência de amostragem de $2 \mathrm{~Hz}$ - Pista de Prova ISO 5008 (2002) acidentada, com velocidade de $15 \mathrm{~km} \mathrm{~h}^{-1}$. Analysis of the FFT with a sampling frequency of $2 \mathrm{~Hz}$ - Track Test ISO 5008 bumpy with a speed of $15 \mathrm{~km} \mathrm{~h}^{-1}$.

É possível observar, pela análise da Figura 15, que a suspensão Twin-Link acompanha o sinal de entrada até a frequência de $0,2 \mathrm{~Hz}$. Isto significa que esta suspensão satisfaz o critério de 
acompanhar a entrada em baixas frequências. Nessa situação, a barra do pulverizador consegue ficar paralela ao quadro do pulverizador, o que corresponde a melhor qualidade de aplicação de defensivo (RAMON et al., 1997). Já a suspensão pendular não consegue acompanhar o quadro do pulverizador, causando maior erro durante a aplicação de defensivo.

\section{CONCLUSÕES}

Pode-se notar, através das várias análises feitas com a transformada rápida de Fourier (FFT), que a suspensão do tipo pêndulo simples tem melhor desempenho que a suspensão trapezoidal quando a frequência de excitação da pista é maior que $2 \mathrm{~Hz}$, independentemente do tipo de pista, suavizada ou acidentada, e do grau de velocidade de translado do pulverizador, $5 \mathrm{~km} \mathrm{~h}^{-1} \mathrm{e}$ $15 \mathrm{~km} \mathrm{~h}^{-1}$.

Por outro lado, quando a frequência de excitação é menor que $0,2 \mathrm{~Hz}$, a suspensão do tipo trapezoidal apresenta melhor desempenho que a suspensão pendular simples, uma vez que, nestas circunstâncias, é desejável que a barra do pulverizador acompanhe as oscilações do chassi. Nessa situação, a barra do pulverizador sempre ficará paralela ao solo, conseguindo, desta maneira, melhor qualidade de aplicação de defensivo.

\section{REFERÊNCIAS}

ANTHONIS, J.; AUDENAERT, J.; RAMON, H. Design optimisation for the vertical suspension of a crop sprayer boom. Biosystems Engineering, London, v.90, n.2, p.153-160, 2005.

DOEBELIN, E. O. Control system principles and design. Montreal: John Wiley \& Sons, 1985.

FROST, A.R.O.; SULLIVAN, J.A. Verification and use of a mathematical model of an active twin link boom suspension. Journal of Agricultural Engineering Research, Silsoe, v.40, p.259-274, 1998.

GÖHLICH, H. Deposition and penetration of spray. To appear in Symposium on application and biology. BCPC Monogram, v.28, p.172-182, 1985.

ISO 5008. Agricultural wheeled tractors and field machinery measurement of whole-body vibration of the operator. 2002.

LARDOUX, Y.; SINFORT, C.; ENFÄLT, P.; MIRALLES, A.; SEVILA, F. Test method for boom suspension influence on spray distribution. Part I: Experimental Study of Pesticide Application under a Moving Boom. Biosystems Engineering, London, v.96, n.1, p.29-39, 2007a.

LARDOUX, Y.; SINFORT, C.; ENFÄLT, P.; MIRALLES, A.; SEVILA, F. Test method for boom suspension influence on spray distribution. Part II: Validation and Use of a Spray Distribution Model. Biosystems Engineering, London, v.96, n.2, p.161-168, $2007 \mathrm{~b}$.

NATION, H.J. The design and performance of a gimbal-type mounting for sprayer booms. 1. Development procedure. Journal of Agricultural Engineering Research, London, v.36, p.233-246, 1987.

O'SULLIVAN, J.A. Simulation of the behaviour of a spray boom with an active and passive pendulum suspension. Journal of Agricultural Engineering Research, London, v.35, p.157-173, 1986.

O'SULLIVAN, J.A. Verification of passive and active versions of a mathematical model of a pendulum spray boom suspension. Journal of Agricultural Engineering Research, London, v.40, p.89-101, 1998.

OZKAN, H.E.; REICHARD, D.L. Effect of orifice wear on flow rate, spray pattern and droplet size distributions of fan pattern nozzles. In: INTERNATIONAL SYMPOSIUM ON PESTICIDES APPLICATION TECHNIQUES, 1993, Strasbourg. Proceedings... v.1, p.159-166. 
PONTELLI, C.O. Comportamento dinâmico de suspensões passivas de barra para pulverizadores. Dissertação (Mestrado) - Bauru, 2007.

RAMON, H.; MISSOTTEN, B.; DE BAERDEMAEKER, J. Spray boom motions and spray distribution, part 2: experimental validation of the mathematical relation and simulation results. Journal of Agricultural Engineering Research, London, v.66, p.31-39, 1997.

SERNEELS, F.; DUCATTILLON, C. Compte Rendu d'Essai "Field trials on efficiency". Paris: Centre Agronomique de Recherches Apliquées du Hainaut a.s.b., 1993. Internal Document. 